\title{
Progression of myopathology in Kearns-Sayre syndrome: a morphological follow-up study*
}

\author{
Heinz Reichmann, R. Gold, B. Meurers, M. Naumann, P. Seibel, U. Walter, and T. Klopstock \\ Department of Neurology, University of Würzburg, Josef-Schneider-Strasse 11, W-8700 Würzburg, Germany
}

Received September 21, 1992/Revised December 29, 1992/Accepted January 14, 1993

Summary. We report on the progression of myopathology by comparing two biopsies from a patient with a Kearns-Sayre-Syndrome. The first biopsy was taken in 1979 and showed 10\% ragged-red fibers. Myopathic changes were slight including internal nuclei and fiber splitting in $10 \%$ of the fibers. Electron microscopy revealed typical mitochondrial abnormalities with regard to number and shape. In 1989 a second biopsy was performed for an extended analysis of mitochondrial DNA. This time less than $5 \%$ of all fibers were ragged-red. Severe myopathic changes could be detected which so far has rarely been reported in mitochondrial cytopathy.

Key words: Myopathy - Kearns-Sayre-syndrome - Cytochrome $c$ oxidase deficiency

The morphological hallmarks for the diagnosis of mitochondrial myopathies are the so called "ragged-red" fibers and ultrastructural changes of mitochondria such as a proliferation and concentric arrangement of cristae, and paracrystalline inclusions [3]. Myopathic features such as an increased variability of fiber size, fiber atrophy, fiber type predominance or deficiency, internal nuclei, and motheaten fibers are usually slight [4].

We report here a progression of muscle pathology in a 53-year-old male patient with Kearns-Sayre syndrome and partial cytochroma $c$ oxidase deficiency, who showed a marked increase of muscle degeneration after a 10-year follow-up. Clinically this period was characterized by a more rapid decline of muscle strength and the development of new symptoms.

\footnotetext{
* Supported by the Deutsche Forschungsgemeinschaft $(\operatorname{Re} 265 / 6-1$ and $\operatorname{Re} 265 / 7-1$ )
}

Correspondence to: H. Reichmann (address see above)

\section{Case report}

In 1979 a 53-year-old patient presented with bilateral ptosis, which had developed 6 months previously, and a mild generalized muscle weakness and exercise intolerance since early childhood. The disease was only slowly progressive until the age of 40 , followed by a more rapidly increasing weakness of proximal limb muscles. There was no past medical history and no family history of neuromuscular diseases. In 1979 neurological examination revealed bilateral ptosis, slight external ophthalmoplegia, facial weakness, and BMRC grade 4 weakness of neck, trunk, and proximal limb muscles.

During the 10-year follow-up he developed exercise dyspnea, intermittent tachycardia, diabetes mellitus, and mild siowly progressive ataxia. Muscle strength had declined to grade 3/4. Besides an initially raised creatine phosphokinase level to $191 \mathrm{U} / \mathrm{l}$ (normal $<80 \mathrm{U} / \mathrm{l}$ ) all routine laboratory examinations revealed normal values. Serum lactate under resting conditions was normal, whereas in CSF lactate was raised to $2.8 \mathrm{mmol} / \mathrm{l}$ (nor$\mathrm{mal}<2.1 \mathrm{mmol} / \mathrm{l})$. CSF total protein was not elevated. Fundoscopy showed pigmentary degeneration of the retina. Because of intermittent tachycardia and exercise dyspnea an extensive cardiological examination was performed. Rest, exercise, and long-term ECG as well as echocardiography and coronary arteriography showed no abnormalities. The electron microscopic examination of a heart muscle biopsy showed abnormal mitochondria with concentric cristae. Initially EMG showed a pure myopathic and later on mixed myopathic and neurogenic pattern. In 1989 the initially normal nerve conduction velocity of the right tibial nerve was reduced to $39 \mathrm{~m} / \mathrm{s}$.

\section{Biopsy findings}

The histological, histochemical, electron microscopic, and biochemical methods are described elsewhere $[2,5]$. A first muscle biopsy (right biceps brachii) was performed in 1979 and confirmed the clinically suspected diagnosis of mitochondrial myopathy. The modified Gomori trichrome stain (Fig. 1a) showed 10\% raggedred fibers. Some of the fibers were conspicuous for extensive vaculolation.

Myopathic changes were only slight and consisted of a mild focal proliferation of endomysium, central nuclei in $10 \%$ of the fibers and fiber splittings. In addition, H\&E stains disclosed a few regenerating fibers (not shown). Morphometric analysis of 200 fibers of each type revealed mild atrophy of type 1 fibers (atrophy factor 470; Fig. 2) and mild hypertrophy of type 2 fibers (hyper- 

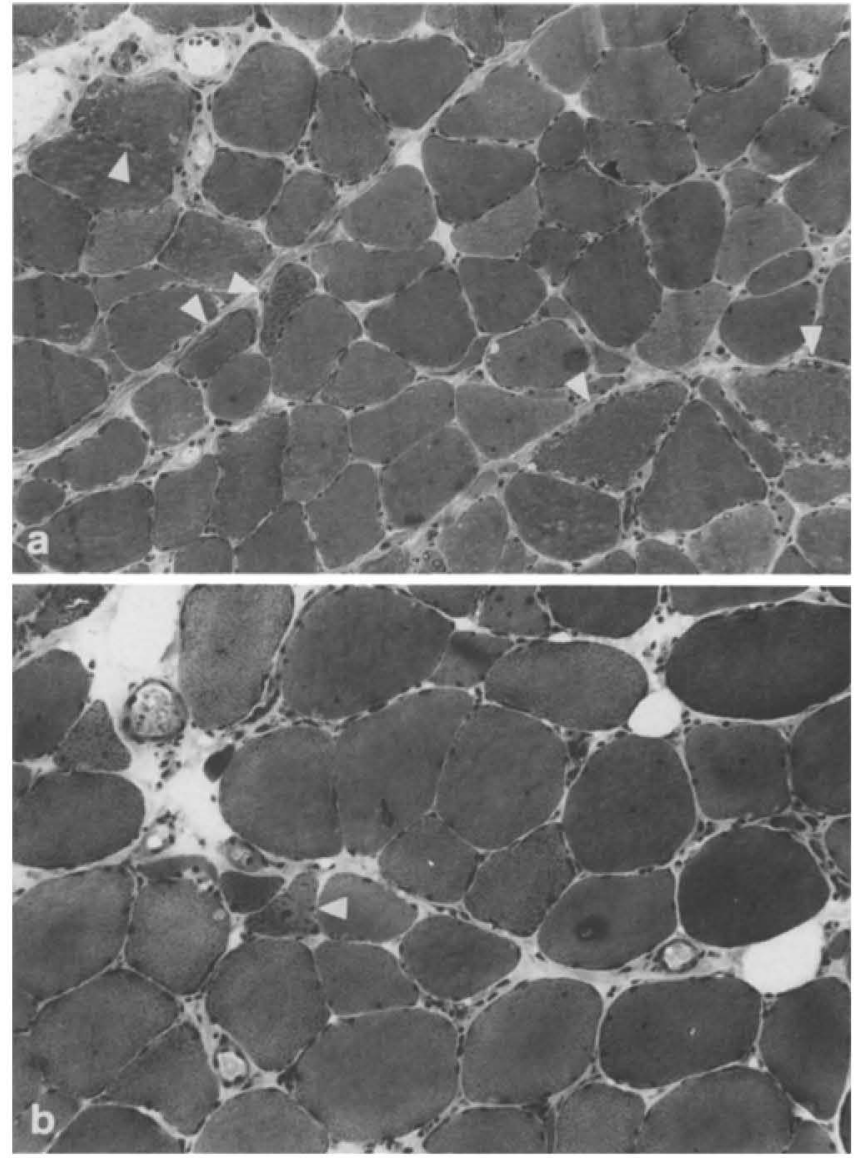

Fig. 1. In 1979 fibers with the typical ragged-red appearance and vacuolar inclusions were present (a; arrowheads); 10 years later mitochondrial proliferation was conspicuous only in atrophic fibers (b, arrowhead). Furthermore, a marked variability of fiber size diameter is present. Modified Gomori trichrome, $\times 140$

trophy factor 750). Atrophy and hypertrophy factors were calculated by a computerized system following the criteria of Dubowitz and Brooke [1]. Electron microscopic examinations revealed abnormal mitochondria with a variety of structural alterations, such as enlarged mitochondria with concentric lamellated cristae and paracrystalline inclusions (Fig. 3a).
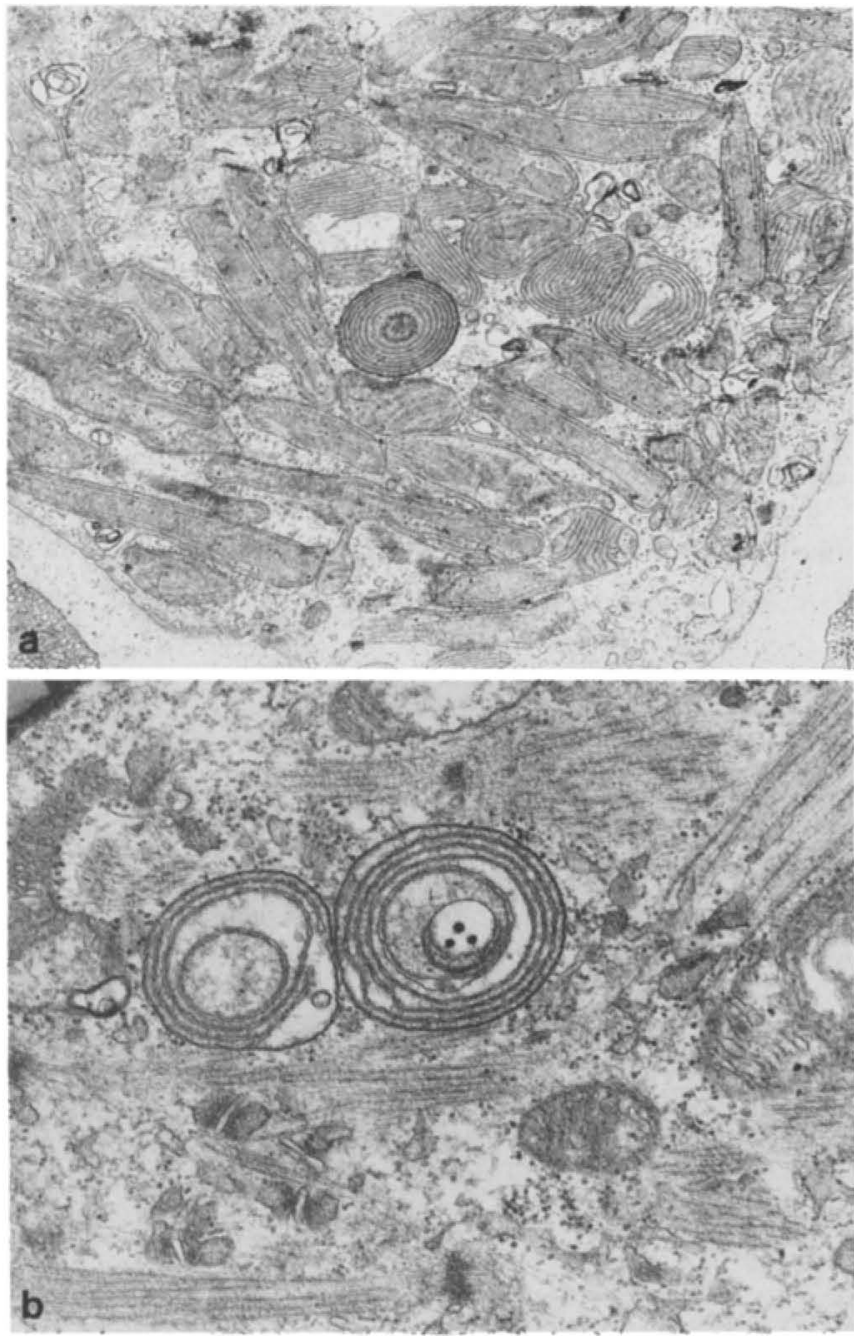

Fig. 3. Electron microscopy revealing abnormal mitochondria with paracrystalline inclusions and hypertrophic concentric lamellae in 1979 (a). In 1989 structural abnormalities in mitochondria are less marked $(\mathbf{b})$. $\mathbf{a} \times 23000 ; \mathbf{b} \times 45000$

\section{Pathological changes in type 1 fibres}

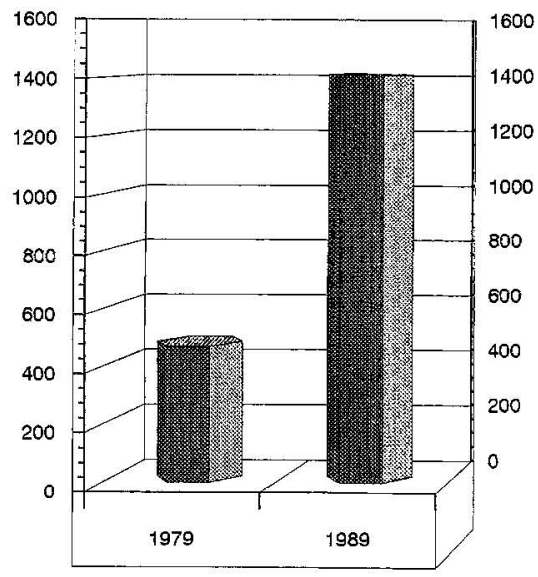

atrophy factors

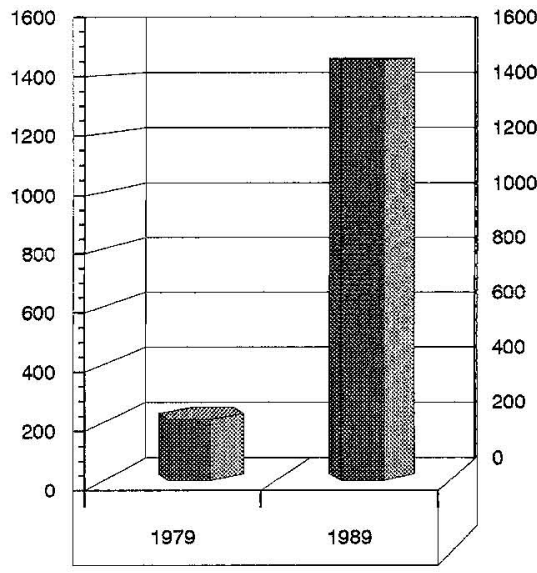

hypertrophy factors
Fig. 2. Morphometric analysis of atrophy and hypertrophy factors in type 1 fibers. Analysis of the second biopsy reveals marked progression of atrophy and strong hypertrophy 
In 1989 a second biopsy (right rectus femoris) was performed for biochemical reasons and for analysis of the mitochondrial genome. The informed consent of the patient was obtained. Again the modified Gomori (Fig. 1b) showed aggregation of mitochon dria, but only in less than $5 \%$ of the fibers. Vacuolation of fibers could no longer be seen. By electron microscopy abnormal mitochondria with concentric lamellated or rarified cristae were only visible in atrophic fibers (Fig. 3b). Paracrystalline inclusions were now absent. Furthermore, moderate to severe myopathic changes could be seen. They consisted of small groups of fat cells, some acute fiber necroses, internal nuclei in about $20 \%$ of fibers and pyknotic nuclear clumps. There was a highly increased variation of fiber size, which was especially pronounced in type 1 fibers (Fig. 2): atrophy and hypertrophy factors were now 1410 and 1455, respectively. Type 2 fibers also underwent strong atrophy (atrophy factor 1180) but only medium-grade hypertrophy (hypertrophy factor 1020). Secondary alterations of muscle fiber membrane composition were excluded by normal dystrophin (Fig. 4) and spectrin immunocytochemistry (not shown).

Histochemical stainings revealed a partial cytochrome $c$ oxidase deficiency in about $80 \%$ of fibers, which could be confirmed by biochemical examinations. The cytochrome $c$ oxidase value was $1.6 \mathrm{U} / \mathrm{g}$ muscle (normal: 1.73-5.0 U/g muscle). Non-collagen muscle protein was normal.

Analyses of the mitochondrial genome were performed on the 1989 biopsy using the Southern blot technique. In addition to the $16.5-\mathrm{kb}$ band only faint bands could be detected, indicating multiple deletions of mitochondrial DNA (data not shown).

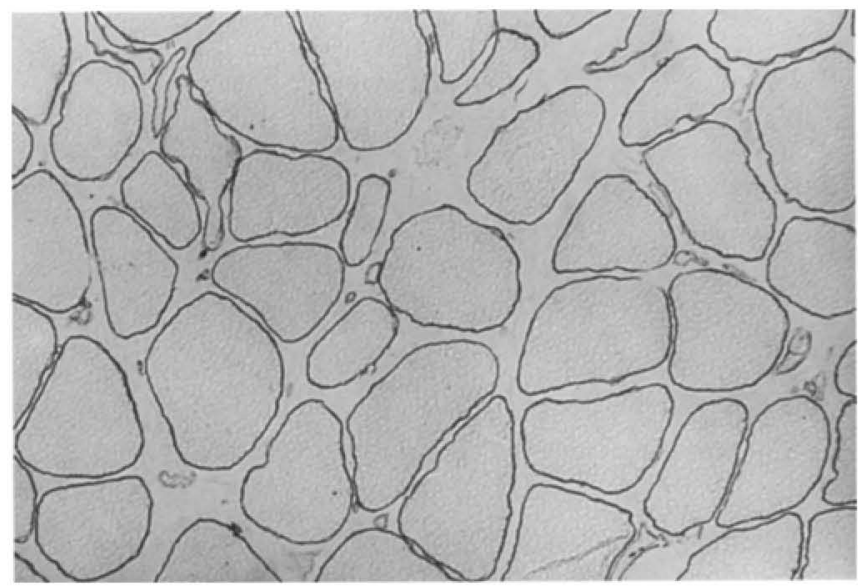

Fig. 4. Immuncytochemical detection of the $C$ terminus of dystrophin shows normal continuous labeling of plasma membranes. $\times 140$

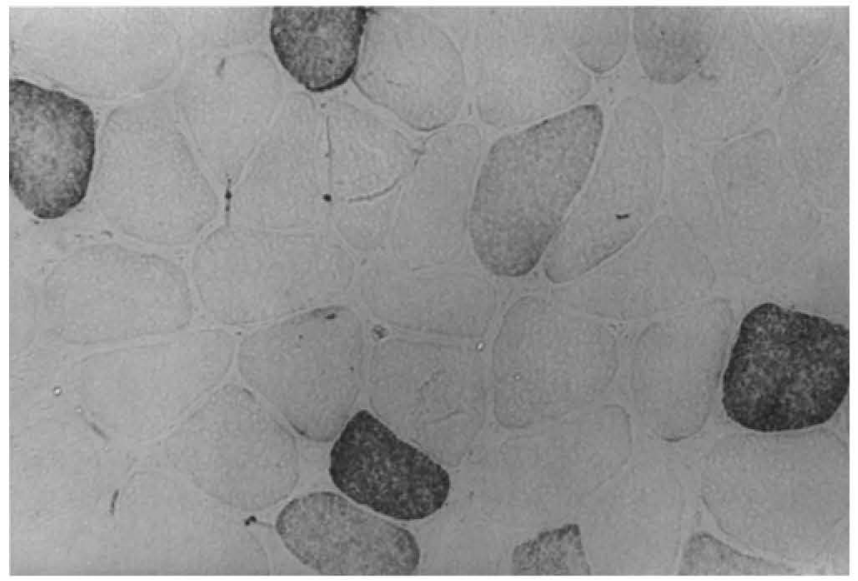

Fig. 5. Cytochrome $c$ oxidase staining shows that about $80 \%$ of the fibers are negative, some fibers have partial deficiency (arrowhead). $\times 140$

\section{Discussion}

With an age of onset before 20 years, progressive external ophthalmoplegia, pigmentary retinopathy and ataxia, our patient fulfilled the criteria of Kearns-Sayre syndrome [1]. After a disease duration of about 45 years muscle pathology showed, in addition to the typical mitochondrial abnormalities, marked myopathic changes. In the literature only a few reports exist about severe myopathic abnormalities in mitochondrial myopathy $[1,4]$. In contrast to our case all of these patients were children below the age of 12 years. Regardless this significant age difference all patients had a fatal or at least severely disabling course of disease.

In comparison to the first 35 years of the disease our patient noticed a more rapid clinical decline during the last 10 years. Morphologically this progression coincided with an outstanding increase of myopathic muscle degeneration and a decrease of mitochondrial abnormalities. The number of ragged-red fibers had fallen from $10 \%$ to less than $5 \%$, and ultrastructural changes of mitochondria became rare. Although the second biopsy had been taken from a different muscle, the influence of the biopsy site is probably of minor importance as clinical affliction of both muscles was comparable in 1989. Furthermore, pathological alterations affected both fiber types, thus reducing a possible bias by a different fiber-type composition of these muscles.

One could speculate that the labile energy status of the mitochondria in ragged-red fibers led to high-grade atrophy or necrosis of these fibers with subsequent replacement by connective tissue and fat. Thus, only fibers whose mitochondria exhibited minor alternations survived.

Acknowledgements. The authors gratefully acknowledge the expert technical assistance of Mrs. S. Seufert and expert secretarial work by Mrs. B. Goebel.

\section{References}

1. Dubowitz V (1985) Muscle biopsy. A practical approach, 2nd edn. Ballière Tindall, London, pp 504-536

2. Moraes CT, DiMauro S, et al (1989) Mitochondrial DNA deletions in progressive external ophthalmoplegia and KearnsSayre syndrome. N Engl J Med 320:1293-1299

3. Morgan-Hughes JA (1976) The mitochondrial myopathies. In: Engel AG, Banker DQ (eds) Myology. Basic and clinical. McGraw-Hill, New York, pp 1709-1743

4. Nonaka I, Koga Y, Shikura K, et al (1988) Muscle pathology in cytochrome $c$ oxidase deficiency. Acta Neuropathol $77: 152-160$

5. Reichmann H, Rohkamm R, Zeviani M, et al (1986) Mitochondrial myopathy due to complex III deficiency with normal reducible cytochrome $c$ concentration. Arch Neurol 43:957-961 